\title{
A Comparative Clinical Study of Oral Clonidine Versus Intravenous Clonidine on Haemodynamic Changes due to Laryngoscopy and Endotracheal Intubation
}

\author{
Sanjeev Singh ${ }^{1,2}$ \\ ${ }^{1}$ Department of Anaesthesiology and Intensive Care, School of Medical Sciences, College of Health Sciences, Kwame Nkrumah University \\ of Science and Technology, Kumasi, Ghana \\ ${ }^{2}$ Department of Anaesthesia and Intensive Care, Satyabhama Academy of Medical Sciences \& Research Institute, Lucknow, India \\ Email address: \\ drsanjeev73@rediffmail.com
}

\section{To cite this article:}

Sanjeev Singh. A Comparative Clinical Study of Oral Clonidine Versus Intravenous Clonidine on Haemodynamic Changes due to Laryngoscopy and Endotracheal Intubation. Pharmaceutical Science and Technology. Vol. 3, No. 2, 2019, pp. 34-39.

doi: $10.11648 /$ j.pst.20190302.11

Received: December 18, 2019; Accepted: December 30, 2019; Published: January 8, 2020

\begin{abstract}
Laryngoscopy with or without endotracheal intubation amounts to a highly noxious stimulus to the haemodynamics of a patient and various efforts have been made to attenuate this response. This study was conducted to compare the effects of oral Clonidine and IV Clonidine premedication on haemodynamic response to laryngoscopy and endotracheal intubation. This is a prospective, randomized controlled, double-blind study conducted after obtaining institutional ethical approval. One hundred normotensive patients between 18-45yrs of age (ASA Grade I \& II) scheduled for elective surgery were sub-divided into two groups with 50 patients in each group. Oral or intravenous clonidine $3 \mu \mathrm{g} / \mathrm{kg}$ was given at 30 and 15 minutes before induction. Patients were Induced with inj Propofol $2.5 \mathrm{mg} / \mathrm{kg}, \mathrm{fentanyl} 1 \mu \mathrm{g} \mathrm{kg}{ }^{-1}$ and inj Vecuronium $0.12 \mathrm{mg} / \mathrm{kg}$ and intubated. Heart rate (HR), systolic blood pressure (SBP), diastolic blood pressure (DBP), rate pressure product (RPP) were recorded at Basal (before administration of study drug), pre laryngoscopy (after administration of study drug but before induction of anaesthesia) and post laryngoscopy at 1, 3, 5, 10 and 15 minutes respectively. HR, SBP, DBP, and RPP decreased by $-3.6 \%,-9.5 \%,-11.5 \%,-12.7 \%$ in the oral clonidine group and by $-3.4 \%,-16.3 \%,-11.9 \%,-19.1 \%$ in IV clonidine group from basal to pre laryngoscopy values $(\mathrm{p}<0.05)$. An increase was seen in both the group at 1,3 and 5 minutes after intubation in both the groups. But the response was significantly less in the IV Clonidine group as compared to the Oral group. All the variables returned towards baseline values by 10-15 minutes post-laryngoscopy in either group. Oral Clonidine $3 \mu / \mathrm{kg}$ was less effective than IV Clonidine $3 \mu \mathrm{g} / \mathrm{kg}$ in blunting haemodynamic stress response. IV Clonidine premedication effectively blunted stress response to endotracheal intubation in ASA physical status I and II patients without causing adverse reactions.
\end{abstract}

Keywords: Attenuation, Clonidine, Haemodynamic, Intubation, Laryngoscopy

\section{Introduction}

Laryngoscopy and tracheal intubation are the most essential tools of an anaesthesiologist in airway management. Endotracheal intubation has been practised routinely and it is a well-known fact that laryngoscopy induces a cardiovascular stress response characterised by hypertension and tachycardia due to reflex sympathetic stimulation [1]. This increase in blood pressure and heart rate are usually transitory variable and unpredictable lasting for a few minutes. It may be well tolerated in healthy individuals but may be hazardous in patients with hypertension, tachycardia, myocardial infarction, cerebrovascular disease and other complications. Several attempts have been made to attenuate haemodynamic changes which include an increase in blood pressure and heart rate in response to laryngoscopy and endotracheal intubation. Pharmacological approaches involving the use of lidocaine (Manjunath et al., 2008) [2], remifentanil (Kaygusuz et al., 2007) [3], fentanyl (Bostana and Eroglu, 2012) [4], a combination of esmolol and nicardipine (Moon 
et al., 2012) [5], a comparative study between lidocaine, diltiazem and esmolol (Singh et al., 2013) [6] have been utilized to attenuate the pressure responses to laryngoscopy and tracheal intubation. Each of these drugs has a unique advantage and disadvantage in blunting the haemodynamic response to laryngoscopy. Reflex changes in the cardiovascular system are most marked after laryngoscopy and lead to an average increase in arterial blood pressure by $40-50 \%$ and $20 \%$ increase in heart rate (Singh et al., 2012) [1]. This can have deleterious effects on the heart as shown by Stoelting (1978) [7]. In the recent decade, several studies have focused on the use of $\alpha_{2}$-adrenergic agonists to attenuate the haemodynamic response following laryngoscopy and tracheal intubation. Clinically used $\alpha_{2}$-adrenergic agonists are dexmedetomidine and clonidine. They are highly selective alpha2-adrenergic agonists, better hypnotic, sedative, and analgesic. It has been used safely for general anaesthesia, postoperative analgesia and Intrathecal Spinal Anaesthesia (ISA) without any respiratory depression [8]. Previously clonidine was available only as oral preparation which had to be given 60-90 minutes before induction, but now with the advent of intravenous (IV) preparation, the time for premedication can be reduced to 10 minutes pre-induction [9] There is a paucity of literature on studies to control haemodynamic changes during laryngoscopy and endotracheal intubation with IV clonidine as well as comparing the effects of oral and IV clonidine on haemodynamic.

\section{Methods}

Study site and participants: This study was undertaken after obtaining approval by the committee on human research publications and ethics at Satyabhama Academy Of Medical Sciences \& Research Institute, Lucknow. Informed consent was obtained from one hundred patients. The study population consisted of American Society of Anaesthesiologists (ASA) physical status I or II, male and female adults between the ages of 18-65 years scheduled for various elective surgical procedures under general anaesthesia.

\subsection{Study Design}

This study was prospective; randomized and doubleblinded clinical comparison between oral and IV clonidine. The Sample size for the study was one hundred generated using a sample size calculator. The study participants were randomly divided into two groups by a computer-generated randomization table. A study nurse (Person A) who was not involved in the randomisation process prepared the study drugs. Person B monitored the Heart Rate (HR), systolic blood pressure (SBP), diastolic blood pressure (DBP), Mean Arterial Pressure (MAP) with respect to time whilst Person C was responsible for intubation of the patients. Person $\mathrm{A}$ and $\mathrm{C}$ were kept constant throughout the study. Person B, C and the patient were unaware of the drug or placebo to enable double-blinding.

\subsection{Inclusion Criteria}

For the study were ASA class I or II; age range 18-65, oropharyngeal anatomy of Mallampati class I and II and elective surgery performed under general anaesthesia with endotracheal intubation.

\subsection{Exclusion Criteria}

For the study excluded patients who were morbidly obese; patients with cardiovascular disease; Heart rate $<60$ beats per minute (bpm), basal $\mathrm{SBP}<100 \mathrm{mmHg}$ and other conditions such as sepsis, patients showing stressful features during induction and laryngoscopy (bucking, coughing, vomiting). Patients undergoing emergency surgery, pregnant, drug allergies, difficult intubations and intubations in, which total duration of laryngoscopy exceeded 15 seconds were excluded from the study.

\subsection{Pre-anaesthetic Protocol}

The day prior to surgery all patients underwent a preanaesthetic evaluation with special consideration to elicit a history of hypertension, dyspnoea, chest pain, cough, wheezing, convulsions and diabetes mellitus, as well as previous anaesthetic history and drug sensitivity. Information collected included weight, nutritional status, airway assessment by the Mallampatti scoring system; a detailed examination of the respiratory; cardiovascular and central nervous system. Preoperative routine investigations were checked. Patients were advised to fast the night prior to surgery.

\subsection{Anaesthesia Protocol}

After patient identification, a short preoperative history was taken; clinical examination and routine investigations were rechecked in all patients. Study objective and procedure were explained to the participants and written informed consent was obtained from each participant. Intravenous access was secured, and an infusion of Ringer's lactate solution was started. Patients were randomly assigned to receive either $3 \mu \mathrm{g} \mathrm{kg}^{-1}$ orally clonidine or IV clonidine, 30 minutes and 15 minutes respectively before induction of anaesthesia respectively. Oral clonidine or IV clonidine was given in the recovery room. Those received IV clonidine $3 \mu \mathrm{g}$ $\mathrm{kg}^{-1}$ were given placebo orally at 30 minutes and those received oral clonidine $3 \mu \mathrm{g} \mathrm{kg}^{-1}$ were given IV NS 15 minutes before induction. The degree of sedation was graded using the Ramsay sedation scale and patients were shifted to the operating room after which routine non-invasive monitor was applied and vital signs monitored. Midazolam $0.04 \mathrm{mg}$ $\mathrm{kg}^{-1}$ and glycopyrrolate $0.2 \mathrm{mg}$ were administered intravenously as premedication and patients were preoxygenated with four to five breaths of $100 \%$ oxygen. All patients received Injection fentanyl $1 \mu \mathrm{g} \mathrm{kg}^{-1}$ then patients were induced with IV propofol $2.5 \mathrm{mg} \mathrm{kg}^{-1}$ in incremental doses until loss of eyelash reflex occurred, then patient's airway assessed for ventilation and IV vecuronium bromide 
$0.12 \mathrm{mg} \mathrm{kg}^{-1}$ was given over $20 \mathrm{sec}$. Patients were ventilated with oxygen and $1 \%$ isoflurane using IPPV with a fresh gas flow of 6 litres $\min ^{-1}$ by Bain circuit until intubation. About 2 min after IV vecuronium, laryngoscopy was performed with a Macintosh laryngoscope blade and trachea intubated with an appropriate size cuffed endotracheal tube. After confirmation of correct placement of ET tube, anaesthesia was then maintained with $\mathrm{O}_{2}$ and isoflurane. HR, SBP, DBP, MAP, $\mathrm{SpO}_{2}$ (oxygen saturation), and ECG (electrocardiogram) changes were recorded. For our study purpose, we recorded vitals at Basal (before administration of study drug), before and after tracheal intubation at 1, 3, 5, 10 and $15 \mathrm{~min}$. No manipulation like painting and draping the area of operation was allowed till $15 \mathrm{~min}$. Injection fentanyl $1 \mu \mathrm{g} \mathrm{kg}^{-1}$ was repeated before incision and further as per the patient's requirement.

\subsection{Parameters and Statistical Analysis}

Summary statistics of patient gender, age, and weight for both the groups were reported as means \pm standard deviation. Ramsay sedation scale was used to assess the level of sedation in all patients before induction. [8]

1. Patient is anxious and agitated or restless, or both.

2. Patient is co-operative, oriented, and tranquil.

3. Patient responds to commands only.

4. Patient exhibits a brisk response to a light glabellar tap or loud auditory stimulus.

5. Patient exhibits a sluggish response to a light glabellar tap or loud auditory stimulus.

6. Patient exhibits no response.

Patients were also assessed for the side effects. HR, SBP, DBP, and MAP were recorded during monitoring. From the data, RPP was calculated by multiplying heart rate with systolic blood pressure. Patients were also observed for complications like over sedation, hypotension, nausea, hypertension, arrhythmias, and hypoxemia. Haemodynamic variables were represented by mean \pm SD. ANOVA with repeated measures was used to compare the changes in HR, SBP, DBP and MAP. Analyzed data were presented in the form of mean, where the level of significance was given as $\mathrm{p}$ value in a separate column. A p-value of less than 0.05 was taken as significant. Man-Whitney $U$ test was used to analyze the data since the data were not following a normal distribution. Nominal data were compared using the ChiSquare test. The statistical package SPSS 14.0 was used.

\section{Results}

A comparison of the demographic profile of the study is as shown in table 1 . No significant difference was observed in the mean age for oral clonidine $(32.4 \pm 8.3)$ when compared to those in IV clonidine $(31.6 \pm 8.1)$. The male to female percentage were $64 \%, 62 \%$ and $36 \%, 38 \%$ in oral and IV clonidine $(\mathrm{p}>0.05)$. In the oral Clonidine group, the range of weights of the patients was between $32-80 \mathrm{kgs}$ with a mean and standard deviation of $52.4 \pm 9.2$ and $55.8 \pm 9.7$ for oral and IV Clonidine groups respectively. No significant difference was observed in the weight distribution in the two groups $(\mathrm{p}>0.05)$.

Table 1. Distribution of patient's demographic profile.

\begin{tabular}{llll}
\hline Variables & $\begin{array}{l}\text { Oral Clonidine }(\mathbf{n}= \\
\mathbf{5 0})\end{array}$ & $\begin{array}{l}\text { IV Clonidine }(\mathbf{n}= \\
\mathbf{5 0})\end{array}$ & P-value \\
\hline Age $($ Yrs $)$ & $32.4 \pm 8.3$ & $31.6 \pm 8.1$ & 0.841 \\
Weight $(\mathrm{Kg})$ & $52.4 \pm 9.2$ & $55.8 \pm 9.7$ & 0.637 \\
Sex M/F & $32(64 \%) / 18(36 \%)$ & $31(62 \%) / 19(38 \%)$ & 0.325 \\
\hline
\end{tabular}

Data are presented as means \pm standard deviation, ratio and percentages. $\mathrm{Kg}=$ kilogram, $\mathrm{Yrs}=$ years, $\mathrm{F}=$ female, $\mathrm{M}=$ male, $\mathrm{P}$ is significant $<0.05$.

The range of the Ramsay sedation score was 2-5 in the oral and IV clonidine groups. The highest Ramsay sedation score of 2 was seen $64 \%$ and $66 \%$ with no significant difference in the oral and IV clonidine groups $(\mathrm{P}>0.05)$. Lower incidence of obvious side effects like severe bradycardia, hypotension and nausea were noticed in $2 \%, 10 \%, 8 \%$ and $4 \%, 2 \%, 6 \%$ in oral and IV clonidine groups respectively as seen in table 2 . Hypotension was statistically significant in oral clonidine group $(\mathrm{P}<0.05)$.

Table 2. Shows the clinical assessment in the IV and oral clonidine groups.

\begin{tabular}{llll}
\hline clinical assessment & Oral Clonidine & IV Clonidine & P-value \\
\hline Ramsay Sedation score 2 & $32(64 \%)$ & $33(66 \%)$ & NS \\
Ramsay Sedation score 3 & $12(24 \%)$ & $13(26 \%)$ & NS \\
Ramsay Sedation score 4 & $2(4 \%)$ & $1(2 \%)$ & NS \\
Ramsay Sedation score 5 & $4(8 \%)$ & $3(6 \%)$ & NS \\
Bradycardia & $1(2 \%)$ & $2(4 \%)$ & NS \\
Hypotension & $5(10 \%)$ & $1(2 \%)$ & $*$ \\
Nausea & $4(8 \%)$ & $3(6 \%)$ & NS \\
\hline
\end{tabular}

Data are presented as percentages NS- Not significant $(\mathrm{P}>0.05), \mathrm{P}$ *Significant; **highly significant.

1. There were total of three patients presented with bradycardia given injection atropine.

2. There were total of six patients presented with hypotension received injection phenylephrin.

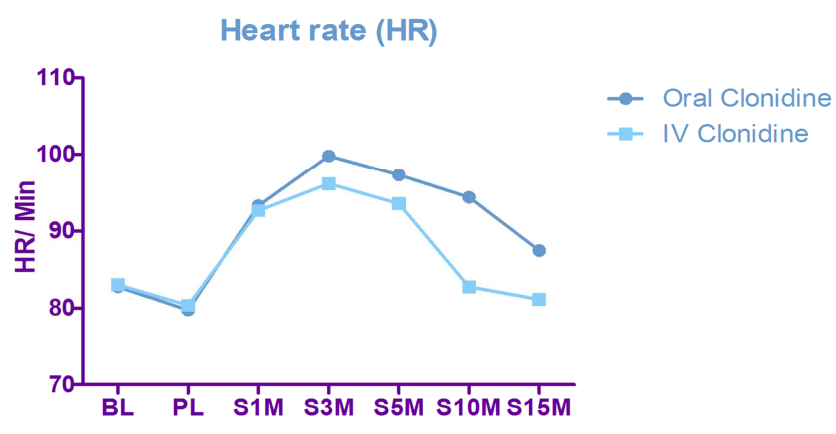

$\mathrm{BL}=\mathrm{B}$ asal; $\mathrm{PL}=$ Pre laryngoscopy; $\mathrm{S}=$ Post laryngoscopy; $\mathrm{M}=\mathrm{M}$ inute

Figure 1. Change in heart rate (HR) in the two study groups.

Basal heart rate (HR) before receiving clonidine was $82.78 \pm 6.47$ and $83.10 \pm 7.53$ in the oral and IV clonidine groups, which is statistically insignificant with P-value $>$ 0.05. Pre laryngoscopy HR was $79.75 \pm 7.21$ and $80.35 \pm 7.96$ with percentage change of $-3.6 \%$ and $-3.4 \%$ in oral clonidine and IV clonidine from the basal values $(\mathrm{P}<0.05)$. HR was well controlled in the IV group as compared to oral clonidine group. There was an increase in HR post laryngoscopy at 1, 5, 10 and 15 minutes as shown in table 3 and figure 1. 
Table 3. Change in heart rate/min (HR) in the two study groups.

\begin{tabular}{llllll}
\hline Parameter & Oral clonidine & \% change & IV clonidine & \% change & P-value \\
\hline Basal & $82.78 \pm 6.47$ & - & $83.10 \pm 7.53$ & - & NS \\
Pre laryngoscopy & $79.75 \pm 7.21$ & $-3.6 \%$ & $80.35 \pm 7.96$ & $-3.4 \%$ & $*$ \\
Post laryngoscopy 1Min & $93.25 \pm 7.35$ & $12.7 \%$ & $92.70 \pm 8.16$ & $11.5 \%$ & $*$ \\
3 Minutes & $99.79 \pm 7.49$ & $20.5 \%$ & $96.24 \pm 7.50$ & $15.8 \%$ & $* *$ \\
5 Minutes & $97.25 \pm 7.53$ & $17.5 \%$ & $93.64 \pm 7.12$ & $12.6 \%$ & $*$ \\
10 Minutes & $94.36 \pm 7.29$ & $14 \%$ & $82.80 \pm 8.19$ & $-0.4 \%$ & $*$ \\
15 Minutes & $87.49 \pm 7.62$ & $5.7 \%$ & $81.17 \pm 8.73$ & $-2.4 \%$ & $* *$ \\
\hline
\end{tabular}

Data are presented as means \pm standard deviation and percentage changes in haemodynamic variables from baseline, P-*Significant; ** highly significant; NS Not significant $(>0.05)$, Min=Minute.

Systolic blood pressure (SBP) decreased by $-9.5 \%$ in the oral clonidine group and by $-16.3 \%$ in IV clonidine group from basal to pre laryngoscopy values $(\mathrm{p}<0.05)$. An increase in the SBP was seen in both the group at $1,3,5$, and 10 minutes after intubation. There was a significant decrease in SBP after 1, 3, 5 and 10 minutes of intubation $(p<0.05)$. In IV clonidine group decrease in SBP was well controlled as compared to oral clonidine group as shown in table 4 and figure 2.

Table 4. Change in systolic blood pressure (SBP) $\mathrm{mmHg}$ in the two study groups.

\begin{tabular}{lllll}
\hline Parameter & Oral clonidine & \% change & IV clonidine & \% change \\
\hline Basal & $124.63 \pm 6.53$ & - & $121.18 \pm 18.06$ & - \\
Pre laryngoscopy & $112.83 \pm 6.38$ & $-9.5 \%$ & $101.30 \pm 10.64$ & $-16.3 \%$ \\
Post laryngoscopy 1Min & $139.89 \pm 7.17$ & $12.2 \%$ & $127.56 \pm 9.95$ & $5.3 \%$ \\
3 Minutes & $140.15 \pm 8.36$ & $12.4 \%$ & $132.26 \pm 9.21$ & $9.2 \%$ \\
5 Minutes & $130.62 \pm 7.06$ & $4.8 \%$ & $128.92 \pm 9.14$ & $6.4 \%$ \\
10 Minutes & $127.94 \pm 7.52$ & $2.6 \%$ & $124.69 \pm 9.70$ & $2.8 \%$ \\
15 Minutes & $126.81 \pm 7.59$ & $1.8 \%$ & $120.86 \pm 9.65$ & $-0.2 \%$ \\
\hline
\end{tabular}

Data are presented as means \pm standard deviation and percentage changes in haemodynamic variables from baseline, P-*Significant; ** highly significant; NS Not significant $(>0.05), \mathrm{mmHg}$ - millimetre of mercury.

Systolic blood pressure $(\mathrm{mm} \mathrm{Hg})$

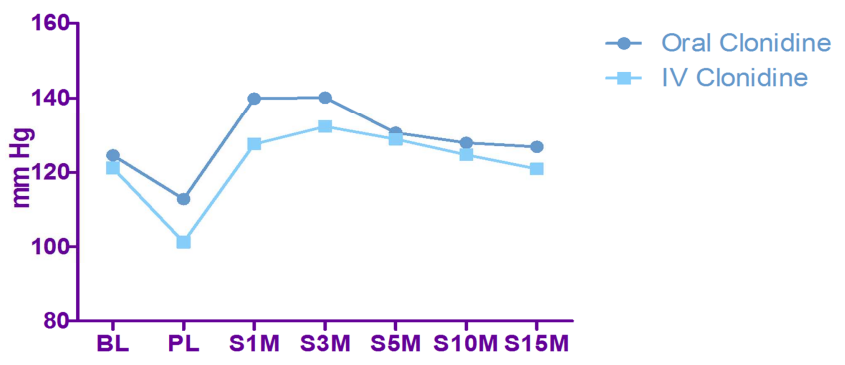

$\mathrm{BL}=\mathrm{B}$ asal; $\mathrm{PL}=$ Pre laryngoscopy; $\mathrm{S}=$ Post laryngoscopy; $\mathrm{M}=\mathrm{M}$ inute

Figure 2. Change in systolic blood pressure (SBP) in two groups.

Diastolic blood pressure (DBP) decreased by $-11.5 \%$ in the oral clonidine group and by $-11.9 \%$ in IV clonidine group from basal to pre laryngoscopy values $(\mathrm{p}<0.05)$. An increase in the DBP was seen in both the group at 1,3 and 5 minutes after intubation. There was a significant decrease in DBP after $1,3,5,10$ and 15 minutes of intubation in the IV clonidine group as compared to oral clonidine group as shown in table 5 and figure 3.

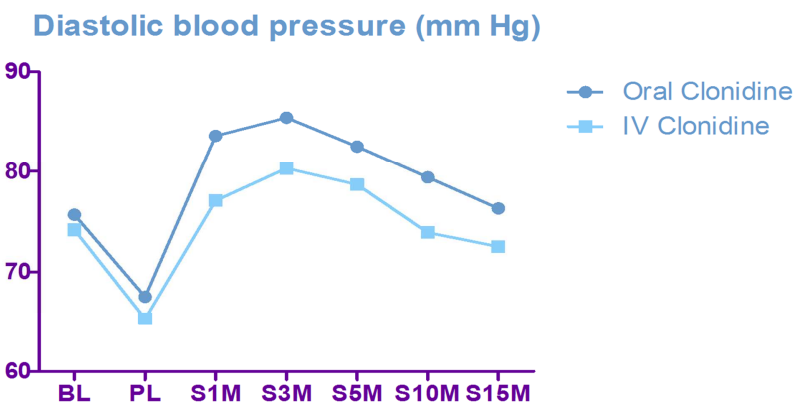

$\mathrm{BL}=\mathrm{B}$ asal; $\mathrm{PL}=$ Pre laryngoscopy; $\mathrm{S}=$ Post laryngoscopy; $\mathrm{M}=\mathrm{M}$ inute

Figure 3. Change in diastolic blood pressure in two groups.

Table 5. Change in diastolic blood pressure (DBP) $\mathrm{mmHg}$ in the two study groups.

\begin{tabular}{lllll}
\hline Parameter & Oral clonidine & \% change & IV clonidine & \% change \\
\hline Basal & $75.82 \pm 5.71$ & - & $74.61 \pm 6.04$ & - \\
Pre laryngoscopy & $67.18 \pm 4.38$ & $-11.5 \%$ & $65.74 \pm 5.63$ & $-11.9 \%$ \\
Post laryngoscopy 1Min & $83.27 \pm 5.15$ & $9.7 \%$ & $77.63 \pm 6.35$ & $4 \%$ \\
3 Minutes & $85.31 \pm 6.38$ & $12.5 \%$ & $80.27 \pm 5.61$ & $7.5 \%$ \\
5 Minutes & $82.57 \pm 5.05$ & $8.8 \%$ & $78.94 \pm 5.84$ & $5.7 \%$ \\
10 Minutes & $79.14 \pm 5.51$ & $4.3 \%$ & $73.57 \pm 5.70$ & $-1.5 \%$ \\
15 Minutes & $76.42 \pm 5.29$ & $0.8 \%$ & $72.86 \pm 6.15$ & $-2.4 \%$ \\
\hline
\end{tabular}

Data are presented as means \pm standard deviation and percentage changes in haemodynamic variables from baseline, P-*Significant; $* *$ highly significant; NS Not significant $(>0.05)$, mmHg- millimetre of mercury. 
Rate pressure product (RPP) decreased by $-12.7 \%$ in the oral clonidine group and by $-19.1 \%$ in IV clonidine group from basal to pre laryngoscopy values $(p<0.05)$. An increase in the RPP was seen in both the group at 1, 3 and 10 minutes after intubation. There was a significant decrease in RPP after $1,3,5$ and 10 minutes of intubation in the IV clonidine group as compared to oral clonidine group as shown in table 6 and figure 4.

Table 6. Change in rate pressure product (RPP) in the two study groups.

\begin{tabular}{|c|c|c|c|c|c|}
\hline Parameter & Oral clonidine & $\%$ change & IV clonidine & $\%$ change & P-value \\
\hline Basal & $10316 \pm 1485$ & - & $10063 \pm 1691$ & - & NS \\
\hline Pre laryngoscopy & $9001 \pm 1252$ & $-12.7 \%$ & $8144 \pm 1158$ & $-19.1 \%$ & $* *$ \\
\hline Post laryngoscopy $1 \mathrm{Min}$ & $13043 \pm 1328$ & $26.4 \%$ & $11819 \pm 1421$ & $17.5 \%$ & $* *$ \\
\hline 3 Minutes & $13981 \pm 1742$ & $35.5 \%$ & $12717 \pm 1385$ & $26.4 \%$ & $* *$ \\
\hline 5 Minutes & $12707 \pm 1571$ & $23.2 \%$ & $12065 \pm 1541$ & $19.9 \%$ & $* *$ \\
\hline 10 Minutes & $12073 \pm 1928$ & $17.1 \%$ & $10316 \pm 1432$ & $2.5 \%$ & $*$ \\
\hline 15 Minutes & $11095 \pm 1841$ & $7.6 \%$ & $9808 \pm 1292$ & $-2.6 \%$ & NS \\
\hline
\end{tabular}

Data are presented as means \pm standard deviation and percentage changes in haemodynamic variables from baseline, P_*Significant; $* *$ highly significant; NS Not significant $(>0.05)$, mmHg- millimetre of mercury.

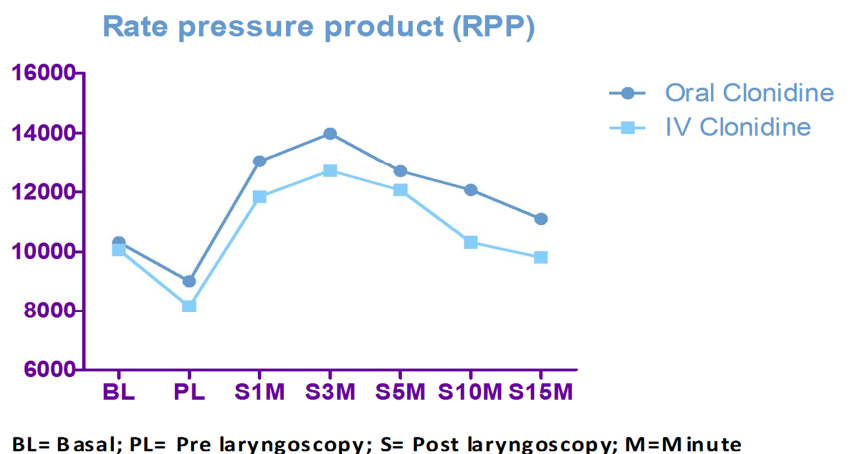

Figure 4. Change in rate pressure product in two groups.

\section{Discussion}

Intubation is associated with a cardiovascular response of elevated systolic blood pressure (SBP) and heart rate (HR), occasional arrhythmias, ischaemia, increased intracranial pressure and intraocular pressure if no specific measures are taken to prevent these haemodynamic responses. The HR and SBP can increase up to $20 \%$ and $50 \%$ respectively depending upon the method of induction [10].

Studies have shown that there is an increased incidence of myocardial infarction when intraoperative heart rates are $>110$ beats per min (bpm) [11]. In our study, none of the patients in the two study groups showed a heart rate $>110$ bpm. RPP is a good estimate of myocardial oxygen requirement. The RPP levels close to 20,000 are normally associated with angina and myocardial ischemia. [6] RPP 1 min after intubation remained less than 20,000 that is 13043 and 11819 in oral and IV clonidine groups. This finding confirms the cardioprotective effect of study drug during laryngoscopy and intubation.

Recently there has been considerable research in the $\alpha 2$ adrenergic agonist group of drugs on attenuating cardiovascular to endotracheal intubation. They act on medulla oblongata at receptor site and presynaptically at peripheral nerve terminals, to cause a reduction in the activity of the sympathetic nervous system [8]. Clonidine, an imidazole compound is a selective agonist for $\alpha 2$ adrenoceptors with a ratio of 200:1 ( $\alpha 2: \alpha 1)$. Though it is primarily an antihypertensive, clonidine has been increasingly used for premedication [8, 12]. Its central action reduces sympathetic activity and stimulates parasympathetic outflow, increasing vagal tone contributing to the slowing of $\mathrm{HR}$, producing sedation, anxiolysis, dryness of secretions with a reduction in requirement of anaesthetic agents and improved haemodynamic stability during laryngoscopy and intubation [12]. Within the last decade, several studies have reported the successful use of oral clonidine premedication to prevent hyperadrenergic and hyperdynamic cardiovascular responses to endotracheal intubation [12]. However, there are not many studies comparing intravenous and oral applications of clonidine prior to induction of anaesthesia. In our study, the intravenous clonidine administration immediately prior to induction of anaesthesia was compared with the more conventional oral clonidine administration for attenuation of haemodynamic response due to laryngoscopy and endotracheal intubation. Both groups were premedicated and anaesthetized similarly. Results of our study indicate that IV clonidine very effectively blunts the stress response due to endotracheal intubation compared to oral clonidine. We chose to use oral clonidine $3 \mu \mathrm{g} / \mathrm{kg}$ because larger doses $(0.2 \mathrm{mg})$ of oral clonidine are known to induce hypotension and bradycardia [13]. The effects of oral clonidine on haemodynamic variables and sedation are dose-dependent. If increasing the dose to more than $4 \mu \mathrm{g} / \mathrm{kg}$ not further enhance efficacy [14]. IV clonidine changes in HR and blood pressure were doses related up to a dose of $4 \mu \mathrm{g} / \mathrm{kg}$, increasing the dose to $6 \mu \mathrm{g} / \mathrm{kg}$ did not enhance the haemodynamic effects and a dose of $2 \mu \mathrm{g} / \mathrm{kg}$ was found to be as placebo [14]. Hence we preferred to use a dose of $3 \mu \mathrm{g} / \mathrm{kg}$ for oral and IV group as considering the high bioavailability of oral clonidine (7095\%) [15]. IV clonidine provided better protection of haemodynamic in response due to endotracheal intubation than did the oral clonidine. However, conclusions concerning dose efficacy relationship cannot be drawn from our data, because plasma concentrations of the oral and IV clonidine were not compared. Our study parameters proved a clinical and statistical significance of intravenous over oral clonidine.

There was a significant decrease in pre laryngoscopy mean heart rate (HR), mean systolic blood pressure (SBP), mean 
diastolic pressure (DBP) and mean rate pressure product (RPP) from basal values (before study drug was administered) in both the groups. After laryngoscopy and intubation rise in IV clonidine was not as significant as oral clonidine group. During the entire operative procedure, all parameters were well controlled with IV clonidine. On analysing these observations, it can be concluded that Clonidine reduces the systolic as well as diastolic blood pressure in the operative period. These findings were in agreement with the studies conducted by Lambert et al. [15] \& Raval et al. [16]. These observations strongly reflect the hypotensive effects of Clonidine and its ability to attenuate the rise in HR and blood pressure during laryngoscopy and intubation.

In comparison to the usual oral clonidine premedication, IV clonidine premedication with $3 \mu \mathrm{g} / \mathrm{kg}$ appeared to be more effective, and regarding the lack of adverse reactions, at least as safe and easy to apply. IV clonidine optimized haemodynamic stability during the induction of anaesthesia, laryngoscopy, intubation, and postoperatively.

\section{Conclusion}

Oral clonidine is though simple and cost-effective, it is less effective in blunting haemodynamic response to endotracheal intubation. Intravenous premedication with $3 \mu \mathrm{g} / \mathrm{kg}$ clonidine, when compared to the traditional oral clonidine, effectively and safely attenuates the haemodynamic response to laryngoscopy and endotracheal intubation without any deleterious effect.

\section{Conflict of Interest}

All the authors do not have any possible conflicts of interest.

\section{Acknowledgements}

Thanks to Dr. Sharad Kumar Govil for his moral support.

\section{References}

[1] Singh, S., Laing, E. F., Ansah-Owiredu, W. K. B., Singh, A., and Annamalai, A. (2013). A study of the efficacy of cardiac antidysrhythmic drugs in attenuating haemodynamic responses to laryngoscopy and endotracheal intubation in the black population. J Anesthe Clinic Res, 4: 326-32.

[2] Manjunath, H. G., Venkatesh, G. S., Prima, V., Jennifer, L. V., and Sathees, B. C. (2008). Can calcium and sodium channel blockers attenuate hemodynamic responses to endotracheal intubation? Eur J Gen Med, 5: 198-207.

[3] Kaygusuz, K., Toker, M. I., Kol, I. O., Erdogan, H., Gursoy, S., and Mimaroglu, C. (2007). The effects of different doses of remifentanil on intraocular pressure after tracheal intuba-tion: a randomized, double-blind and pro-spective study. Ann Ophthalmol (Skokie), 39: 198-204.
[4] Bostana, H., and Eroglu, A. (2012). Comparison of the clinical efficacies of fentanyl, esmolol and lidocaine in preventing the hemody-namic responses to endotracheal intubation and extubation. Journal of Current Surgery, 2: 24-28.

[5] Moon, Y. E., Lee, S. H., and Lee, J. (2012). The optimal dose of esmolol and nicardipine for mantaining cardiovascular stability during rapid sequence induction. Journal of Clinical Anaesthesia, 24: 8-13.

[6] Singh, S., Laing, E. F., Ansah-Owiredu, W. K. B., and Singh, A. (2012). Attenuation of cardiovascular response by $\beta$ blocker esmolol during laryngoscopy and intubation. Journal of Medical and Biomedical Sciences, 1 (4): 27-33.

[7] Stoelting, R. K. (1978). Blood Pressure and heart rate changes during short duration laryngoscopy for tracheal intubation: Influence of viscous or intravenous lidocaine. Anesth Analg, 57: 197-99.

[8] Annamalai, A., Singh, S., Singh, A., and Mahrous, D. E. (2013). Can intravenous dexmedetomidine prolong bupivacaine intrathecal spinal anesthesia? J Anesth Clin Res, 4: 372-77.

[9] Ali, N. P., Kanchi, M., Singh, S., Prasad, A., and Kanase, N. (2014). Dexmedetomedine-ketamine versus propofolketamine as anaesthetic agents in paediatric cardiac catheterization. JAFMC Bangladesh, 10 (1); 19-24.

[10] Singh, S., Kulsum, Shroff, H., Singh, S., Annamalai, A., and Mahrous, D. E. (2013). Can sodium channel blocker lidocaine attenuate haemodynamic responses to endotracheal intubation in patients with coronary artery disease effectively? Journal of Anesthesiology, 1 (3): 27-35.

[11] Singh, S., Laing, E. F., Ansah-Owiredu W. K. B., and Singh, A. (2013). Comparison of esmolol and lidocaine for attenuation of cardiovascular stress response to laryngoscopy and endotracheal intubation in a Ghanaian population. Anesth Essays Res, 7: 83-8.

[12] Allam, J., Sheikh, M. M., and Ara, R. (2018). Effect of oral clonidine on hemodynamic changes due to laryngoscopy, intubation and operative procedures stress response. Arch Phar \& Pharmacol Res, 1 (3). DOI: 10.33552/APPR.2018.01.000512.

[13] Carabine, U. A., Wright, P. M. C., and Moore, J. (1991). Preanaesthetic medication with Clonidine: A dose response study. Br J Anaesth, 67: 79-83.

[14] Kulka, P. J., Tryba, M., and Zenz, M. (1995) Dose response effects of intravenous clonidine on stress response during induction of anaesthesia in CABG patients. Anaesthesia \& Analgesia, 80 (2): 263-268.

[15] Lambert, P., Cyna, A. M., Knight, N., and Middleton, P. (2014). Clonidine premedication for postoperative analgesia in children. Cochrane Database of Systematic Reviews 1, doi.org/10.1002/14651858.CD009633.

[16] Raval, D. L., and Mehta, M. K. (2002). Oral clonidine premedication for attenuation of hemodynamic response to laryngoscopy and intubation. Indian J Anaesth, 46 (2): 124-29. 